\title{
Bloch-type photonic skyrmions in optical chiral multilayers
}

\author{
Qiang Zhang $\odot$, Zhenwei Xie $\odot,{ }^{*}$ Luping Du $\odot,{ }^{\dagger}$ Peng Shi $\odot$, and Xiaocong Yuan ${ }^{\ddagger}$ \\ Nanophotonics Research Center, Shenzhen Key Laboratory of Micro-Scale Optical Information Technology \& Institute of Microscale \\ Optoelectronics, Shenzhen University, Shenzhen 518060, China
}

(Received 16 December 2020; accepted 9 April 2021; published 10 May 2021)

\begin{abstract}
Magnetic skyrmions are topological quasiparticles in magnetization. Recently, as one of their photonic counterparts, Néel-type photonic skyrmions were discovered in evanescent electromagnetic waves. The deep-subwavelength features of the photonic skyrmions suggest their potential in optical imaging quantum technologies and data storage. Here, by exploiting the photonic quantum spin Hall effect of a plasmonic vortex in a trilayered structure, we predict the existence of photonic twisted-Néel- and Bloch-type skyrmions in chiral materials. Their chirality-dependent features can be considered as additional degrees of freedom for future chiral sensing, information processing, and storage technologies. In particular, our findings enrich the formations of photonic skyrmions and reveal a remarkable resemblance of the feature of chiral materials in two seemingly distant fields: photonic skyrmions and magnetic skyrmions.
\end{abstract}

DOI: 10.1103/PhysRevResearch.3.023109

\section{INTRODUCTION}

Skyrmions, hedgehogs of electron spins, are topologically stable magnetization swirls in magnetic materials. Typically, they are defined as two-dimensional spin textures with nonzero topological winding numbers stablized at magnetic fields [1,2]. Their ultracompact size and unique topological protection properties make skyrmions promising for future applications in topological spintronics, information processing, and data storage [3-7] to name but a few. There are two typical magnetic skyrmions: Néel-type and Bloch-type skyrmions [8-15]. Until recently, the photonic counterpart of magnetic skyrmions has been discovered in surface plasmon polaritons (SPPs) $[16,17]$. To date, only the Néel-type photonic skyrmion has been predicted and observed [16-21].

In common optical materials (isotropic, nonmagnetic, and nonchiral), SPPs solely exist for transverse magnetic (TM) polarization [22]. They are confined at the interface between a metal and a dielectic and exponentially decay in the perpendicular direction into the materials. SPPs carry transverse spin angular momentum (SAM) that is perpendicular to its momentum (propagation) direction due to the quantum spin Hall effect (QSHE) of light [23]. For a plasmonic optical vortex $(\mathrm{OV})$ beam at the metal-air interface, the transverse SAM $\left(S_{r}\right)$ is perpendicular to the azimuthal direction of energy flux and thereby orients radially, as shown in Fig. 1(a).

\footnotetext{
*ayst3_1415926@sina.com

$\dagger$ lpdu@szu.edu.cn

†xyuan@szu.edu.cn
}

Published by the American Physical Society under the terms of the Creative Commons Attribution 4.0 International license. Further distribution of this work must maintain attribution to the author(s) and the published article's title, journal citation, and DOI.
So, its spin-orbit coupling results in a Néel-type skyrmion photonic structure with the local photon spin vector (that is, the orientation of the SAM) either along or opposite to the orbital angular momentum [17]. The same mechanism applies to the photonic Néel-type skyrmion lattice generated by the interference of plane-wave SPPs [16,18]. So far, both twisted Néel and Bloch-type skyrmions have been untapped in the novel domain of photonic skyrmions limited by this drawback. Here we confine our discussion in the scheme of plasmonic $\mathrm{OV}$ and show how a Bloch-type photonic skyrmion can exist in chiral materials (materials that are noncentrosymmetric), and control its swirl handedness by tuning the chirality.

In a plasmonic OV [Fig. 1(a)], a Néel-type skyrmion can be understood according to its spin vector texture in the cylindrical coordinate $\left(S_{r}, S_{\varphi}, S_{z}\right)$, with $S_{\varphi}=0$ and $\chi=0$, where $\chi$ is the helicity angle. By comparing with the Bloch type $\left(0, S_{\varphi}, S_{z}\right)$, one finds immediately that the transverse spin $S_{r}$ should be zero. The wave vector of a surface wave (evanescent wave or SPPs) is complex and can be written in $\mathbf{k}=\mathbf{k}_{\mathbf{t}}+i \mathbf{k}_{\mathbf{n}}$ ( $\mathbf{k}_{\mathbf{t}}$ is tangential to the surface and related to the momentum, and $\mathbf{k}_{\mathbf{n}}$ normal to the surface and related to the decay). The transversality condition $\mathbf{E} \cdot \mathbf{k}=0$ necessarily requires that the orthogonal components of the electric field vector in the propagation plane always have a $\pi / 2$ phase difference and thereby generate the transverse spin $\left(S_{r}\right)$ due to the cycloidal rotation. It was shown that $S_{r} \propto$ Rek $\times \operatorname{Imk}$ [23], which indicates that the momentum (propogation) direction, the decay direction, and the transverse spin direction of a SPP constitutes a right-handed system. This is the so-called spin-momentum locking [23-28]. Notice that the SAM (S= $\left.\frac{1}{4 \omega} \operatorname{Im}\left[\epsilon_{c} \mathbf{E}^{*} \times \mathbf{E}+\mu_{0} \mathbf{H}^{*} \times \mathbf{H}\right]\right)$ continuously evolves in the material, so does the transverse spin $S_{r}$. So, in principal, the zero-value point of $S_{r}$ exists in the case where $S_{r}$ varies from positive to negative. We construct this variation by exploiting the causality requirement and spin-momentum locking. The causality requires that the decay of a surface wave must direct 


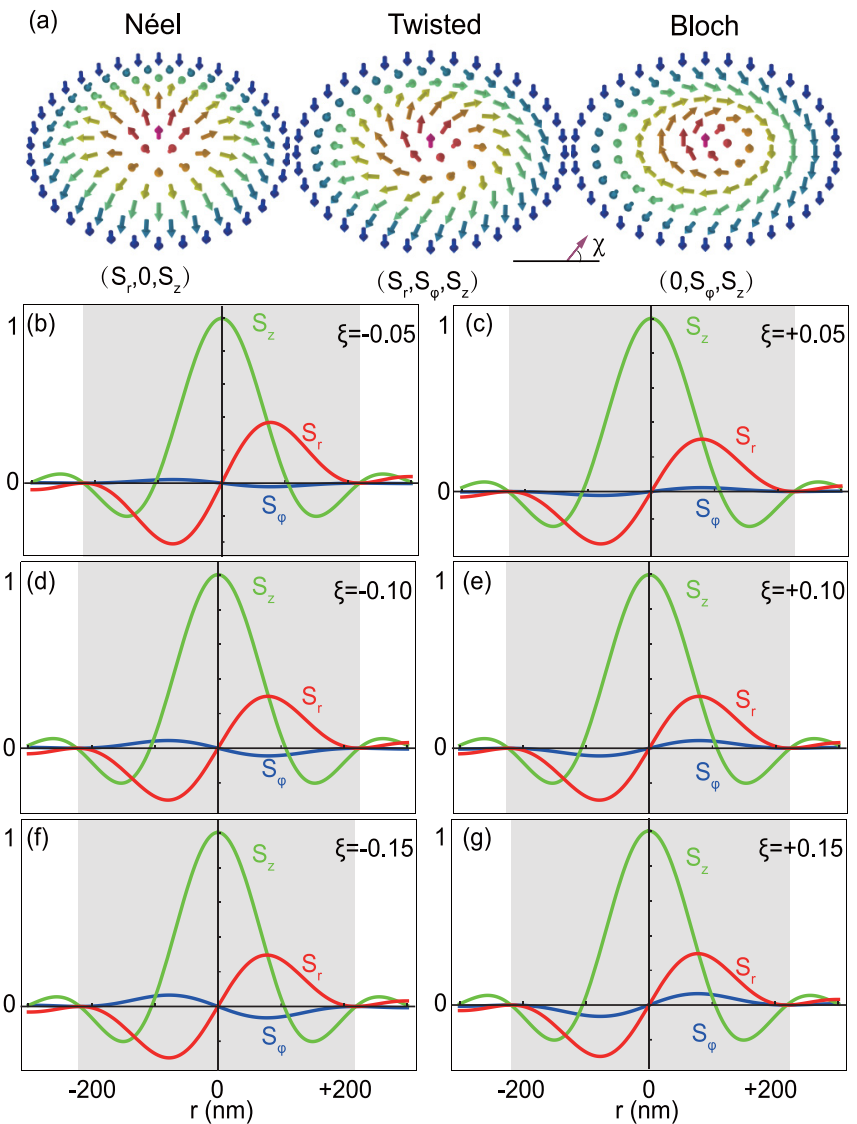

FIG. 1. (a) Photonic skyrmions can be defined according to their SAM vector components, with $\left(S_{r}, 0, S_{z}\right)$ for Néel type $(|\cos \chi|=1)$, $\left(S_{r}, S_{\varphi}, S_{z}\right)$ twisted Néel type $(0<|\cos \chi|<1)$, and $\left(0, S_{\varphi}, S_{z}\right)$ Bloch type $(\cos \chi=0)$. (b)-(g) Evolution of the SAM vector components $S_{r}, S_{\varphi}$ and $S_{z}$ along the radius of the plasmonic-OV in a chiralmetal structure. Gray regions define the photonic skyrmion's size with $r=r_{1}$.

from the boundary toward the material. Hence, a confined structure (e.g., a slab) with its opposite surfaces bounded will introduce antiparallel decay vectors $\mathbf{k}_{\mathbf{n}}$, while the momentum vector $\mathbf{k}_{\mathbf{t}}$ inside is constant. As such, according to the right-handed spin momentum locking, $S_{r}$ flips sign at the two boundaries. Consequently, a zero $S_{r}$ will emerge between the boundaries. In addition, study highlights that in chiral materials, SPPs exhibit hybrid modes of TM and transverse electric polarizations [29]. This offers the opportunity to form a nonzero $S_{\varphi}$. Therefore, we show a Bloch-type photonic skyrmion can be realized in a trilayered metal-insulator-metal (MIM) structure with the introduction of chirality.

\section{TWISTED NÉEL-TYPE PHOTONIC SKYRMIONS}

The electromagnetic response of chiral materials obeys the following constitutive relation [30]: $\mathbf{D}=\epsilon_{c} \mathbf{E}+\mathrm{i} \xi \sqrt{\mu_{0} \epsilon_{0}} \mathbf{H}$, $\mathbf{B}=\mu_{0} \mathbf{H}-\mathrm{i} \xi \sqrt{\mu_{0} \epsilon_{0}} \mathbf{E}$, where $\xi$ and $\epsilon_{c}$ are the chirality parameter and the permittivity of the chiral material, respectively. $k_{ \pm}=\left(\sqrt{\epsilon_{c} / \epsilon_{0}} \pm \xi\right) \omega / c$ are eigenwave numbers corresponding to the right-circularly polarized (RCP) and left-circularly polarized (LCP) waves, respectively. Using
Bohren decompositions [31], $\mathbf{F}_{ \pm}=\mathbf{E} \pm \mathrm{i} \eta \mathbf{H}\left(\eta=\sqrt{\mu_{0} / \epsilon_{c} \epsilon_{0}}\right.$ is the wave impedance of the chiral medium), the field equations can be written as $\nabla \times \mathbf{F}_{ \pm}= \pm k_{ \pm} \mathbf{F}_{ \pm}, \quad \nabla^{2} \mathbf{F}_{ \pm}+$ $k_{ \pm}^{2} \mathbf{F}_{ \pm}=0$, with $\mathbf{E}=\left(\mathbf{F}_{+}+\mathbf{F}_{-}\right) / 2$ and $\mathbf{H}=\left(\mathbf{F}_{+}-\mathbf{F}_{-}\right) / 2 \mathrm{i} \eta$. $\mathbf{F}_{ \pm}$field of a vortex beam in the chiral medium has mode solution as $\mathbf{F}_{ \pm}=\mathbf{f}_{ \pm}(r) \exp [\mathrm{i}(\beta z-\omega t+l \varphi)]$, where $l$ represents the topological charge. In the cylindrical coordinate system, $f_{ \pm z}$ is determined by $\frac{1}{r} \frac{\partial}{\partial r}\left(r \frac{\partial f_{ \pm z}}{\partial r}\right)+\left(k_{ \pm}^{2}-\beta^{2}-\frac{l^{2}}{r^{2}}\right) f_{ \pm z}=0$, which has the solution $f_{ \pm z}=a_{ \pm} J_{l}\left(k_{r} r\right)$. Here $k_{r}^{2}=k_{ \pm}^{2}-\beta^{2}$, $a_{ \pm}$are arbitrary constants representing the field amplitude, and $J_{l}$ is the $l$ th order Bessel function of the first kind. The rest of the field functions are given by the curl of $\mathbf{F}_{ \pm}$. For a plasmonic OV, the in-plane wave-vector component $\left(k_{r}\right)$ is larger than the wave vector $\left(k_{ \pm}\right)$of the beam, resulting in an imaginary axial wave-vector component (denoted as $i k_{+z}$ and $\mathrm{i} k_{-z}$, representing RCP and LCP eigenwaves, respectively). By replacing the $z$-component wave vector in the equations above with the imaginary values $\mathrm{i} k_{+z}$ and $\mathrm{i} k_{-z}$, we can obtain the $f$ fields for a plasmonic $\mathrm{OV}$ in the chiral medium. We can treat the metal in the same way. After obtaining the electromagnetic field solutions in the metal, we get the dispersion relation of the plasmonic $\mathrm{OV}$ from the eigen solution for the boundary condition at the chiral-metal interface (see Appendix A). Subsequently, $k_{r}$ is determined by the dispersion relation and the eigenfield solutions can be resolved. Consequently, the SAM of the plasmonic OV can be calculated as follows:

$$
\mathbf{S}=\left(\begin{array}{c}
S_{r} \\
S_{\varphi} \\
S_{z}
\end{array}\right)=\frac{\epsilon_{c}}{4 \omega} \frac{l}{r}\left(\begin{array}{c}
\frac{J_{l}^{2}}{k_{r}^{2}}\left(a_{+}^{2} k_{+z}+a_{-}^{2} k_{-z}\right) \\
\frac{J_{l}^{2}}{k_{r}^{2}}\left(a_{+}^{2} k_{+}-a_{-}^{2} k_{-}\right) \\
\frac{J_{l} J_{l}^{\prime}}{k_{r}}\left(a_{+}^{2}+a_{-}^{2}\right)
\end{array}\right) .
$$

In the isotropically nonchiral system, SPPs solely exist for TM polarizations and, as a result, $S_{\varphi}$ is constantly zero. Compared to the spin texture expression for air-metal structures [17], we can see that a nonzero $S_{\varphi}$ emerges in Eq. (A1). Most importantly, this term is chirality dependent. According to the reciprocity, a LCP wave in a right-handed system undergoes the same physical response with a RCP wave in a left-handed system. $S_{\varphi}$ is therefore an odd function since $a_{+}^{2} k_{+}$and $a_{-}^{2} k_{-}$ would swap places for opposite chirality in this expression, which is also confirmed by our numerical calculations. We hence plot the spin vector structure's profile of the plasmonic $\mathrm{OV}$ in Fig. 1. Parameters throughout the paper are chosen as follows: $l=1, \lambda=532 \mathrm{~nm}$, Drude permittivity model for gold; for the chiral material $\epsilon_{c}=1.33^{2}$ and $|\xi| \leqslant 0.15$ which can be obtained in a polycrystalline film of chlorophyll $a$ [32]. For $\xi=0$, the spin texture for the SAM is a photonic analog of a Néel-type magnetic skyrmion [12], as shown in Fig. 2(b). For nonzero $\xi$, the spin texture is a photonic analog of a twisted Néel-type magnetic skyrmion [33-36], as shown in Figs. 1(b)-1(g), 2(c), and 2(d).

To further verify the skyrmion analogy, we calculate the skyrmion number (n) of the spin vector structure $\quad n=\frac{1}{4 \pi} \iint \mathbf{M} \cdot\left(\frac{\partial \mathbf{M}}{\partial x} \times \frac{\partial \mathbf{M}}{\partial y}\right) d x d y$, where $\quad \mathbf{M}=$ $(\cos \phi(\alpha) \sin \theta(r), \sin \phi(\alpha) \sin \theta(r), \cos \theta(r)) \quad$ represents the unit vector in the direction of the local SAM vector within the plasmonic OV $(\mathbf{r}=(r \cos \alpha, r \sin \alpha))$, and the integral is taken over a unit cell of the skyrmion in 

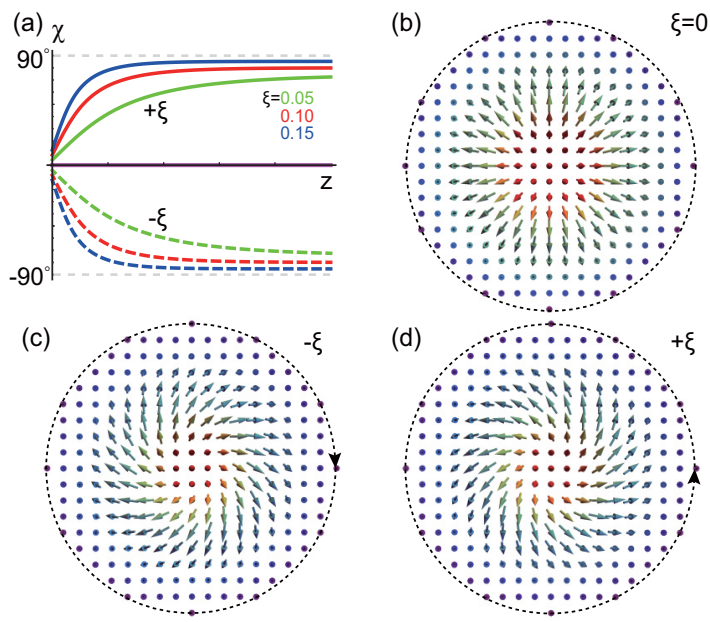

FIG. 2. (a) Helicity angle $(\chi)$ profile as a function of $z$ for the twisted skyrmions with different chirality parameters. $\chi$ is unable to reach $90^{\circ}$ inside the chiral material regardless of its chirality due to the existence of nonzero $S_{r}$. (b)-(d) Illustrations of the skyrmion structures at the interface. The handedness of the twist depends solely on the sign of the material's chirality.

the $X-Y$ plane. Thus, the skyrmion number results in $n=-\frac{1}{4 \pi} \quad[\cos \theta(r)]_{\theta(0)}^{\theta\left(r_{1}\right)}[\phi(\alpha)]_{(\alpha=0)}^{(\alpha=2 \pi)}$. For a twisted spin texture, $\phi(\alpha)=\alpha+\chi$ [11], where $\chi$ is the helicity as defined in Fig. 1(a). Equation (A1) implies that the helicity is independent of the radius as $\chi=S_{\varphi} / S_{r}$. Therefore, for a photonic spin structure with the spin vector changing progressively from the up state $(\cos \theta(0)=1)$ to the down state $\left(\cos \theta\left(r_{1}\right)=-1\right)$ at each domain as shown in the gray zone of Figs. 1(b) $-1(\mathrm{~g})$, the skyrmion number can be evaluated as $n=1$.

At the chiral-metal interface, the plasmonic OV possesses a twisted skyrmion spin texture in analogy to the surface twisted skyrmions in bulk chiral magnets [33-35]. Inside the chiral magnets, Bloch-type magnetic skyrmions with $\chi= \pm 90^{\circ}$ are stable and uniformly stacked along the bulk crystal $[35,36]$. However, it should be stressed that for a chiral plasmonic $\mathrm{OV}$ at a single interface, Bloch-type skyrmions do not exist whatsoever in the chiral material because, regardless of the distance from the chiral surface, a plasmonic OV always carries a nonzero $S_{r}$ component even when the chirality is strong. We illustrate the quantitative evolution of the helicity angle $\chi$ along the $z$ direction of a plasmonic OV in such a chiralmetal structure in Fig. 2(a). $\chi$ exponentially approaches a stable angle as the plasmonic OV decays into the chiral material and eventually cuts off before $\pm 90^{\circ}$. The cutoff angle depends on the chirality. However, the mechanism for the formation of such a twisted photonic skyrmion is prerequisite to the realization of a Bloch-type photonic skyrmion.

\section{BLOCH-TYPE PHOTONIC SKYRMIONS}

Thus far, we have proved that in a single interface structure, the photonic Bloch-type skyrmion does not exist even for systems with strong chirality. Specifically, this is restricted by the intrinsic QSHE of the SPPs [23], that is, the transverse spin $\left(S_{r}\right)$ never vanishes in such a structure. Such effect hinders the
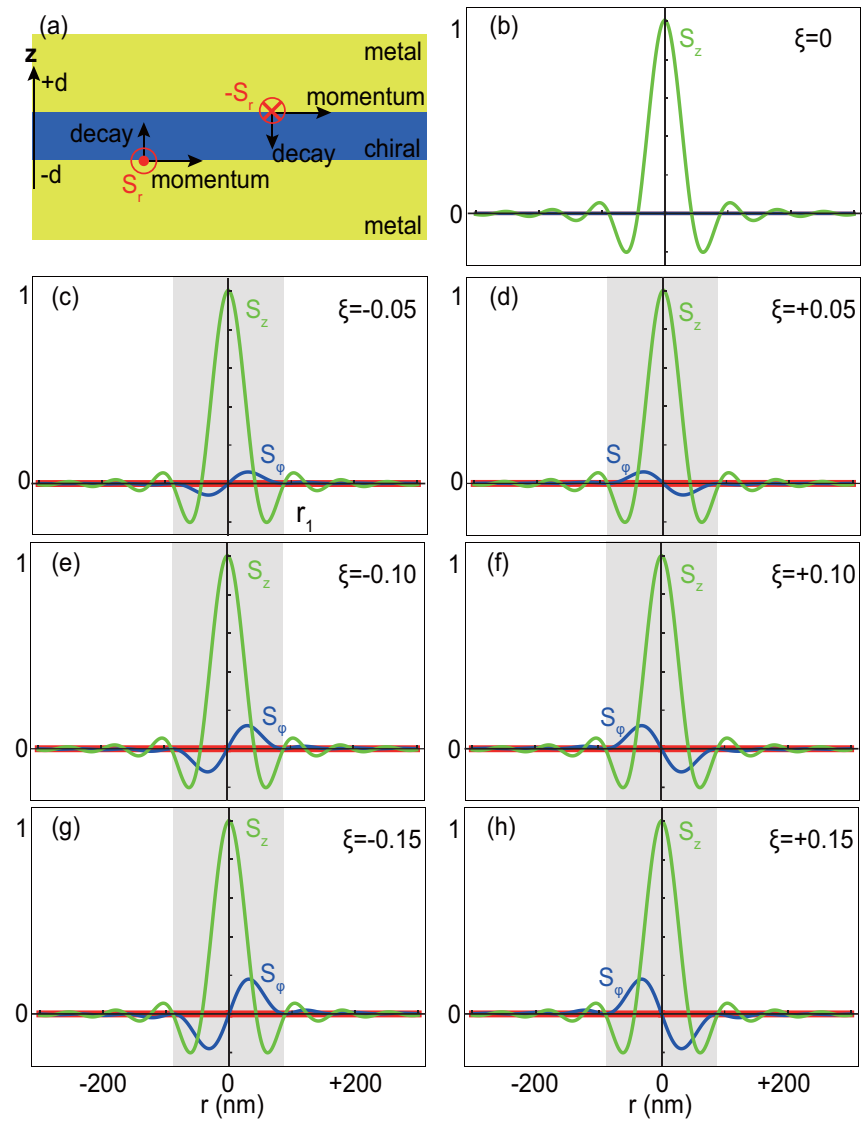

FIG. 3. (a) Schematic illustration of the opposite transverse spins $\left(S_{r}\right)$ on the surfaces of a film that supports coupled plasmons. (b) For a nonchiral system $(\xi=0)$, the SAM in the center of the film only has $S_{z}$ component, so it is not a skyrmion. (c)-(h) $S_{\varphi}$ emerges when the chirality is introduced and depends on the magnitude and sign of $\xi$.

formation of Bloch-type photonic skyrmions. Nevertheless, we can in turn exploit this effect by engineering counter transverse spin structures to extinguish $S_{r}$ while keeping the $S_{\varphi}$ component to realize $\chi= \pm 90^{\circ}$. So in a MIM structure [Fig. 3(a)], the SPPs have transverse spins with opposite signs on the surfaces of the core film. The SAM expression for the plasmonic $\mathrm{OV}$ in such a structure $(-d \leqslant z \leqslant+d)$ is (see Appendix B)

$$
\mathbf{S}=\frac{\epsilon_{c}}{4 \omega} \frac{l}{r}\left(\begin{array}{c}
\frac{J_{l}^{2}}{k_{r}^{2}}\left[\left(-a_{1+}^{2} e^{2 k_{+z}(z-d)}+a_{2+}^{2} e^{-2 k_{+z}(z+d)}\right) k_{+z}\right. \\
\left.-\left(a_{1-}^{2} e^{2 k_{-z}(z-d)}+a_{2-}^{2} e^{-2 k_{-z}(z+d)}\right) k_{-z}\right] \\
\frac{J_{l}^{2}}{k_{r}^{2}}\left[\left(a_{1+} e^{k_{+z}(z-d)}+a_{2+} e^{-k_{+z}(z+d)}\right)^{2} k_{+}\right. \\
\left.-\left(a_{1-} e^{k_{-z}(z-d)}+a_{2-} e^{-k_{-z}(z+d)}\right)^{2} k_{-}\right] \\
\frac{J_{l}^{\prime} J_{l}^{\prime}}{k_{r}^{3}}\left[\left(a_{1+} e^{k_{+z}(z-d)}+a_{2+} e^{-k_{+z}(z+d)}\right)^{2} k_{+}^{2}\right. \\
+\left(a_{1-} e^{k_{-z}(z-d)}+a_{2-} e^{-k_{-z}(z+d)}\right)^{2} k_{-}^{2} \\
+\left(a_{1+} e^{k_{+z}(z-d)}-a_{2+} e^{-k_{+z}(z+d)}\right)^{2} k_{+z}^{2} \\
\left.+\left(a_{1-} e^{k_{-z}(z-d)}-a_{2-} e^{-k_{-z}(z+d)}\right)^{2} k_{-z}^{2}\right]
\end{array}\right) .
$$

When the SPPs decay inside the core, a region of $S_{r}=0$ will emerge somewhere (this region is exactly the center of a symmetric structure, for instance, as in the following dis- 


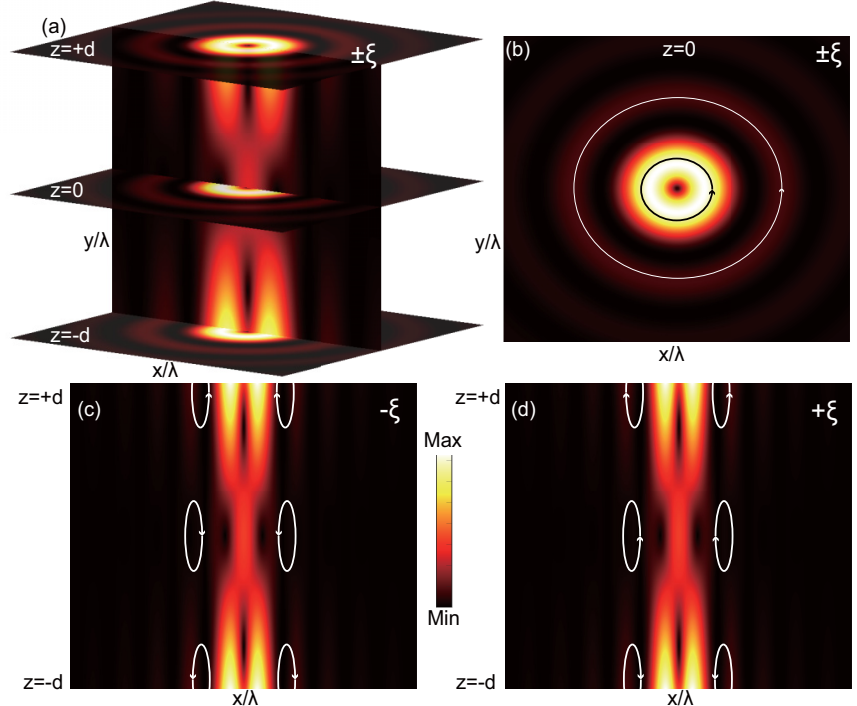

FIG. 4. (a) Energy flux inside the chiral film $(-d \leqslant z \leqslant+d)$. Nonzero $P_{z}$ exists. (b) Azimuthal energy flux is dominant and independent of the material's chirality. (c), (d) $P_{z}$ and $P_{r}$ have reversed signs for $\xi$ with opposite signs, implying the chirality-dependent behavior of $S_{\varphi}$ via $\mathbf{S} \propto \boldsymbol{\nabla} \times \mathbf{P}$.

cussion, an asymmetric structure example can be found in Appendix E). Here we take a core of thickness $2 d=10 \mathrm{~nm}$ as an example, while the conclusion holds consistent for other thicknesses (see Appendix D). For a nonchiral and isotropic material, this results in a plasmonic OV with a spin texture which has solely an $S_{z}$ component in the structure center, as shown in Figs. 3(b) and 5(b). When chirality is introduced, a nonzero $S_{\varphi}$ component appears whose magnitude (sign) are associated with the magnitude (sign) of the material's chirality, see Figs. 3(c)-3(h). It is noted that compared to the single interface case [Fig. 1(h), for instance], the handedness of the Bloch-type skyrmion's swirl [Fig. 3(h), for instance] is reversed for the same material chirality. This results from the strong field coupling between the top and bottom surfaces of the chiral material when it is a very thin film such as $10 \mathrm{~nm}$. The strong coupling magnifies the discrepancy between coefficients $a_{+}$and $a_{-}$associated with the RCP and LCP eigenwaves and thereby reverses the sign of the term $a_{+}^{2} k_{+}-a_{-}^{2} k_{-}$. When the central film thickness is increased, the coupling attenuates and $S_{\varphi}$ behaves like the single interface structure while $S_{r}$ maintains zero in the center (see Appendix D). It is also demonstrated that a more deeply subwavelength skyrmion size $(r \sim 90 \mathrm{~nm}, 1 / 6 \lambda)$ is achieved compared with that $(r \sim 220 \mathrm{~nm})$ in the single interface structure. The compressed effective wavelength by such a highly confined structure contributes to an ultracompact plasmonic OV together with the skyrmion structure.

On the other hand, the Poynting vector (that is, the energy flux density) of the plasmonic OV can be calculated by $\mathbf{P}=$ $\operatorname{Re}\left(\mathbf{E}^{*} \times \mathbf{H}\right) / 2$ (see Appendix $C$ ) and has shown its relation to the $S A M$ via $\mathbf{S} \propto \nabla \times \mathbf{P}[37,38]$. A more close inspection at the energy flux corresponding to the Bloch skyrmions in Figs. 3(g) and 3(h) is plotted in Fig. 4. Inside the chiral film, a nonzero $P_{z}$ component exists [Figs. 4(a), 4(c) and 4(d)] which is distinctively different from the zero plasmonic-OV as in Ref. [17]. Azimuthal energy flux $P_{\varphi}$ still dominates [Fig. 4(b)]. The reversed $P_{z}$ component [Figs. 4(c) and 4(d)] also implies that the azimuth component $\left(S_{\varphi}\right)$ of the SAM exhibits opposite signs for $\pm \xi$.

Finally, we reveal an intriguing resemblance of the characteristics of the photonic skyrmions and magnetic skyrmions in chiral materials. The evolution of the helicity angle of a skyrmion's spin texture along the chiral film thickness is
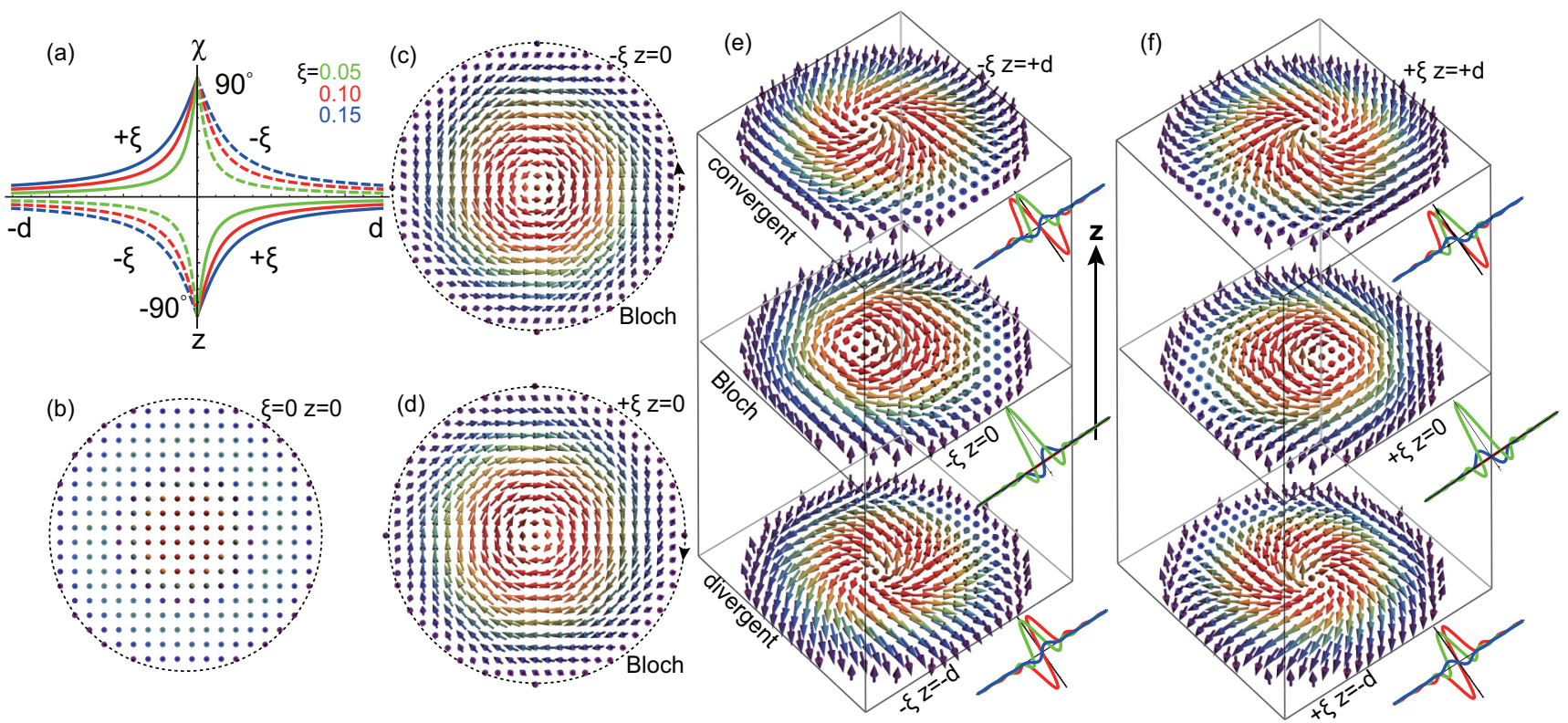

FIG. 5. Illustrations of calculated spin textures. (a) Diagram for the evolution of the helicity angle of the skyrmion's spin texture as a function of the chiral film thickness. (b) SAM vectors with only $S_{z}$ component in the center of a nonchiral film. (c), (d) Bloch-type skyrmion structures with opposite handedness of swirls in the chiral films with opposite chirality, respectively. (e), (f) Twisted skyrmion structures on the bottom and top surfaces of the chiral film, respectively. The insets show the corresponding $\left(S_{r}, S_{\varphi}, S_{z}\right)$ distributions. 
presented in Fig. 5(a). All the representative spin textures can be described from this diagram. For a nonchiral film, the spin vectors in the center are depicted in Fig. 5(b). The photonic skyrmions in a plasmonic chiral multilayerd structure (three-layered metal-chiral-metal structure in this work) possess numerous similarities with the magnetic skyrmions in chiral magnets $[35,36]$ as follows: On the top and bottom surfaces of a chiral film, divergent and convergent [Figs. 5(e) and 5(f)] twisted Néel-type skyrmions are formed, respectively, and eventually the twisted skyrmion evolves into a Bloch-type skyrmion in the chiral film center, with its swirl handedness solely depends on the film's chirality [Figs. 5(c) and 5(d)].

\section{DISCUSSION AND CONCLUSION}

In summary, the presented study enlarges the family of photonic skyrmions. We have demonstrated theoretically the existence of a Bloch-type photonic skyrmion. Without loss of generality, we first take a chiral-metal plasmonic interface as an example and show how a twisted Néel-type photonic skyrmion structure is formed in a plasmonic OV. The twist of the skyrmion's swirl handedness is determined by the magnitude and sign of the material's chirality. Based on this finding, we subsequently present the mechanism of extinguishing the transverse SAM $\left(S_{r}\right)$ of the plasmonic OV inside a threelayered metal-chiral-metal structure. The Bloch-type photonic skyrmion is predicted in this structure and the skyrmion size is greatly minimized compared to the single-interface structures. The swirl handedness of Bloch-type photonic skyrmions is determined by the magnitude and sign of the material's chirality simultaneously. This property implies a possible chiral sensing scheme in optics. In magnetics, enhanced coupling of magnetic skyrmions can be achieved in multilayers with successive ultrathin Co layers $[39,40]$. We note that, based on our theory, it is also possible to further enhance the $S_{\varphi}$ component by exploring multilayered thin-film plasmonic structures with successive metal-chiral interfaces. We also note that it is possible to find Bloch-type electric skyrmions (like the Néel-type electric skyrmion in Ref. [16]) in structured chiral multilayers relying on the hybrid mode property of chiral SPPs. We show optical chiral materials can be a prototypical candidate to realize a plethora of photonic skyrmion types. The chirality-dependent sense of rotation of Bloch-type photonic skyrmions suggests another element in the toolbox for chiral quantum optics [41] as photonic skyrmions can transfer their topological properties to proximate quantum emmitters. The femtosecond scale creation and annihilation of the nontrivial SAM topology enables us to envisage the application of photonic skyrmions in ultrafast quantum infomation processing. The nanometer scale of local spin variation of photonic skyrmions also allows for optical high-density data storage.

\section{ACKNOWLEDGMENTS}

This research was supported by the National Key R\&D Program of China, Grant No. 2018YFB1801801, Guangdong Major Project of Basic Research No. 2020B0301030009, National Natural Science Foundation of China (Grants No. 61935013, No. U1701661, No. 61975133, No. 11774240, No. 62075139, No. 11604218, and No. 61705135), leadership of Guangdong Province Program Grant No. 00201505, the Natural Science Foundation of Guangdong Province Grants No. 2016A030312010, No. 2020A1515011185, the Science and Technology Innovation Commission of Shenzhen Grants No. RCJC20200714114435063, No. KQJSCX20170727100838364, No. ZDSYS201703031605029, No. JCYJ20180507182035270, and No. JCYJ20200109114018750, Shenzhen Peacock Plan (Grant No. KQTD20170330110444030), and Shenzhen University (Grant No. 2019075). Q.Z. also acknowledges the funding support by the National Natural Science Foundation of China (Grant No. 12047540).

\section{APPENDIX A: DISPERSION FOR PLASMONIC OPTICAL VORTEX AT A CHIRAL-METAL STRUCTURE}

$\mathbf{F}_{ \pm}$field of a vortex beam in the chiral medium has a mode solution as

$$
\mathbf{F}_{ \pm}=\mathbf{f}_{ \pm}(r) \exp [\mathrm{i}(\beta z-\omega t+l \varphi)],
$$

where $l$ represents the topological charge. In the cylindrical coordinate system,

$$
\begin{gathered}
\nabla \times \mathbf{F}_{ \pm}= \pm k_{ \pm} \mathbf{F}_{ \pm}, \\
\nabla^{2} \mathbf{F}_{ \pm}+k_{ \pm}^{2} \mathbf{F}_{ \pm}=0,
\end{gathered}
$$

can be written as

$$
\begin{aligned}
\nabla^{2} F_{ \pm r}-\frac{2}{r^{2}} \frac{\partial F_{ \pm \varphi}}{\partial \varphi}-\frac{F_{ \pm r}}{r^{2}}+k_{ \pm}^{2} F_{ \pm r} & =0, \\
\nabla^{2} F_{ \pm \varphi}+\frac{2}{r^{2}} \frac{\partial F_{ \pm r}}{\partial \varphi}-\frac{F_{ \pm \varphi}}{r^{2}}+k_{ \pm}^{2} F_{ \pm \varphi} & =0, \\
\nabla^{2} F_{ \pm z}+k_{ \pm}^{2} F_{ \pm z} & =0 .
\end{aligned}
$$

Substituting Eq. (A1) into Eq. (A3c), we can obtain

$$
\frac{1}{r} \frac{\partial}{\partial r}\left(r \frac{\partial f_{ \pm z}}{\partial r}\right)+\left(k_{ \pm}^{2}-\beta^{2}-\frac{l^{2}}{r^{2}}\right) f_{ \pm z}=0,
$$

which is a Bessel function with general solution $\left(e^{\mathrm{i} l \varphi} e^{-\mathrm{i} \omega t} e^{\mathrm{i} \beta z}\right.$ is omitted)

$$
f_{ \pm z}=a_{ \pm} J_{l}\left(k_{r} r\right),
$$

where $k_{r}^{2}=k_{ \pm}^{2}-\beta^{2}, a_{ \pm}$is arbitrary constant and $J_{l}$ is the $l$ th order Bessel function of the first kind.

Combining with Eq. (A2), we get

$$
\begin{aligned}
\frac{1}{r} \frac{\partial f_{ \pm z}}{\partial \varphi}-\frac{\partial f_{ \pm \varphi}}{\partial z} & = \pm k_{ \pm} f_{ \pm r}, \\
\frac{\partial f_{ \pm r}}{\partial z}-\frac{\partial f_{ \pm z}}{\partial r} & = \pm k_{ \pm} f_{ \pm \varphi}, \\
\frac{1}{r}\left[\frac{\partial}{\partial r}\left(r f_{ \pm \varphi}\right)-\frac{\partial f_{ \pm r}}{\partial \varphi}\right] & = \pm k_{ \pm} f_{ \pm z} .
\end{aligned}
$$

For a plasmonic OV, the in-plane wave-vector component $\left(k_{r}\right)$ is larger than the wave vector $\left(k_{ \pm}\right)$of the beam, resulting in an imaginary axial wave-vector component (denoted as $\mathrm{i} k_{+z}$ and $\mathrm{i} k_{-z}$, representing RCP and LCP, respectively). By replacing the $z$-component wave vector in the equations above 
with the imaginary values $\mathrm{i} k_{+z}$ and $\mathrm{i} k_{-z}$ and based on

$$
\begin{aligned}
& \mathbf{E}=\frac{\mathbf{F}_{+}+\mathbf{F}_{-}}{2}, \\
& \mathbf{H}=\frac{\mathbf{F}_{+}-\mathbf{F}_{-}}{2 \mathrm{i} \eta},
\end{aligned}
$$

the electromagnetic fields for a plasmonic OV (when $z=0$ ) can be derived as

$$
\begin{aligned}
& E_{r}=\frac{1}{2}\left[\frac{\mathrm{i} l}{r} \frac{J_{l}}{k_{r}^{2}}\left(a_{+} k_{+}-a_{-} k_{-}\right)-\frac{J_{l}^{\prime}}{k_{r}}\left(a_{+} k_{+z}+a_{-} k_{-z}\right)\right], \\
& E_{\varphi}=\frac{1}{2}\left[-\frac{\mathrm{i} l}{r} \frac{J_{l}}{k_{r}^{2}}\left(a_{+} k_{+z}+a_{-} k_{-z}\right)-\frac{J_{l}^{\prime}}{k_{r}}\left(a_{+} k_{+}-a_{-} k_{-}\right)\right], \\
& E_{z}=\frac{1}{2}\left(a_{+}+a_{-}\right) J_{l}, \\
& H_{r}=\frac{1}{2 \eta}\left[\frac{l}{r} \frac{J_{l}}{k_{r}^{2}}\left(a_{+} k_{+}+a_{-} k_{-}\right)+\mathrm{i} \frac{J_{l}^{\prime}}{k_{r}}\left(a_{+} k_{+z}-a_{-} k_{-z}\right)\right], \\
& H_{\varphi}=\frac{1}{2 \eta}\left[-\frac{l}{r} \frac{J_{l}}{k_{r}^{2}}\left(a_{+} k_{+z}-a_{-} k_{-z}\right)+\mathrm{i} \frac{J_{l}^{\prime}}{k_{r}}\left(a_{+} k_{+}+a_{-} k_{-}\right)\right], \\
& H_{z}=\frac{1}{2 \eta}(-\mathrm{i})\left(a_{+}-a_{-}\right) J_{l} .
\end{aligned}
$$

Subsequently, the SAM of the plasmonic OV can be calculated:

$$
\mathbf{S}=\left(\begin{array}{c}
S_{r} \\
S_{\varphi} \\
S_{z}
\end{array}\right)=\frac{\epsilon_{c}}{4 \omega} \frac{l}{r}\left(\begin{array}{c}
\frac{J_{l}^{2}}{k_{r}^{2}}\left(a_{+}^{2} k_{+z}+a_{-}^{2} k_{-z}\right) \\
\frac{J_{l}^{2}}{k_{r}^{2}}\left(a_{+}^{2} k_{+}-a_{-}^{2} k_{-}\right) \\
\frac{J_{l} J_{l}^{\prime}}{k_{r}}\left(a_{+}^{2}+a_{-}^{2}\right)
\end{array}\right) .
$$

So once we know $k_{r}$, everything can be deducted.

Now let us see the plasmonic OV in the metal. Notice that the metal is in the half space that $z<0$, so $\beta$ is replaced by $\left(-\mathrm{i} k_{z}\right)$ in this case. We can get the electromagnetic fields as follows:

$$
\begin{aligned}
E_{r} & =\frac{k_{z}}{k_{r}} B J_{l}^{\prime}-\frac{\omega \mu}{k_{r}^{2}} \frac{l}{r} C J_{l}, \\
E_{\varphi} & =\frac{\mathrm{i} k_{z}}{k_{r}^{2}} \frac{l}{r} B J_{l}-\frac{\mathrm{i} \omega \mu}{k_{r}} C J_{l}^{\prime}, \\
E_{z} & =B J_{l}, \\
H_{r} & =\frac{\omega \varepsilon_{m}}{k_{r}^{2}} \frac{l}{r} B J_{l}+\frac{k_{z}}{k_{r}} C J_{l}^{\prime}, \\
H_{\varphi} & =\frac{\mathrm{i} \omega \varepsilon_{m}}{k_{r}} B J_{l}^{\prime}+\frac{\mathrm{i} k_{z}}{k_{r}^{2}} \frac{l}{r} C J_{l}, \\
H_{z} & =C J_{l} .
\end{aligned}
$$

Therefore, the boundary condition of $E_{r}, E_{\varphi}, H_{r}$, and $H_{\varphi}$ at $z=0$ leads to a $4 \times 4$ matrix as a function of the coefficients $a_{ \pm}, B$, and $C$ as

$$
\left|\begin{array}{cccc}
\mathrm{i} \frac{l}{r} \frac{k_{+}}{k_{r}^{2}} J_{l}-\frac{k_{+z}}{k_{r}} J_{l}^{\prime} & -\mathrm{i} \frac{l}{r} \frac{k_{-}}{k_{r}^{2}} J_{l}-\frac{k_{-z}}{k_{r}} J_{l}^{\prime} & -\frac{k_{z}}{k_{r}} J_{l}^{\prime} & \frac{\omega \mu}{k_{r}^{2}} \frac{l}{r} J_{l} \\
-\mathrm{i} \frac{l}{r} \frac{k_{+z}}{k_{r}^{2}} J_{l}-\frac{k_{+}}{k_{r}} J_{l}^{\prime} & -\mathrm{i} \frac{l}{r} \frac{k_{-z}}{k_{r}^{2}} J_{l}+\frac{k_{-}}{k_{r}} J_{l}^{\prime} & -\mathrm{i} \frac{l}{r} \frac{k_{z}}{k_{r}^{2}} J_{l} & \mathrm{i} \frac{\omega \mu}{k_{r}} J_{l}^{\prime} \\
\frac{1}{2 \eta} \frac{l}{r} \frac{k_{+}}{k_{r}^{2}} J_{l}+\mathrm{i} \frac{1}{2 \eta} \frac{k_{+z}}{k_{r}} J_{l}^{\prime} & \frac{1}{2 \eta} \frac{l}{r} \frac{k_{-}}{k_{r}^{2}} J_{l}-\mathrm{i} \frac{1}{2 \eta} \frac{k_{-z}}{k_{r}} J_{l}^{\prime} & -\frac{l}{r} \frac{\omega \varepsilon_{m}}{k_{r}^{2}} J_{l} & -\frac{k_{z}}{k_{r}} J_{l}^{\prime} \\
-\frac{1}{2 \eta} \frac{l}{r} \frac{k_{+z}}{k_{r}^{2}} J_{l}+\mathrm{i} \frac{1}{2 \eta} \frac{k_{+}}{k_{r}} J_{l}^{\prime} & \frac{1}{2 \eta} \frac{l}{r} \frac{k_{-z}}{k_{r}^{2}} J_{l}+\mathrm{i} \frac{1}{2 \eta} \frac{k_{-}}{k_{r}} J_{l}^{\prime} & -\mathrm{i} \frac{\omega \varepsilon_{m}}{k_{r}} J_{l}^{\prime} & -\mathrm{i} \frac{l}{r} \frac{k_{z}}{k_{r}^{2}} J_{l}
\end{array}\right|=0 .
$$

Consequently, we plot the comparison of the dispersion curves for a nonchiral case and chiral case in Fig. 6, where we take a large value for the chirality parameter to show the difference more clearly. According to the free electron gas model proposed by Drude, the complex permittivity of a metal is given by

$$
\varepsilon_{m}=\left(1-\frac{\omega_{p m}^{2}}{\omega(\omega+i \gamma)}\right) \varepsilon_{0},
$$

where $\omega_{p m}$ is the electron plasma frequency of the metal and $\gamma$ denotes the electron collision frequency. $\gamma=1.08 \times 10^{14}$ $\mathrm{rad} / \mathrm{sec}, \omega_{p m}=1.35 \times 10^{16} \mathrm{rad} / \mathrm{sec}$ for $\mathrm{Au}$ [42]. The exact solution for $k_{r}$ is then obtained numerically.

\section{APPENDIX B: DISPERSION FOR PLASMONIC OV AT A METAL-CHIRAL-METAL STRUCTURE}

In a metal-chiral-metal structure, the $f$ fields for a plasmonic $\mathrm{OV}$ in the chiral medium as $\left(e^{i l \varphi} e^{-i \omega t}\right.$ is omitted) can be written as

$$
\begin{aligned}
f_{+r}= & \left(\frac{\mathrm{i} a_{1+}}{k_{r}}\left(\frac{l}{r} \frac{k_{+}}{k_{r}} J_{l}-\mathrm{i} k_{+z} J_{l}^{\prime}\right) e^{k_{+z}(z-d)}\right) \\
& +\left(\frac{\mathrm{i} a_{2+}}{k_{r}}\left(\frac{l}{r} \frac{k_{+}}{k_{r}} J_{l}+\mathrm{i} k_{+z} J_{l}^{\prime}\right) e^{-k_{+z}(z+d)}\right), \\
f_{+\varphi}= & \left(-\frac{a_{1+}}{k_{r}^{2}}\left(-\mathrm{i} k_{+z} \frac{l}{r} J_{l}+k_{r} k_{+} J_{l}^{\prime}\right) e^{k_{+z}(z-d)}\right) \\
& +\left(-\frac{a_{2+}}{k_{r}^{2}}\left(\mathrm{i} k_{+z} \frac{l}{r} J_{l}+k_{r} k_{+} J_{l}^{\prime}\right) e^{-k_{+z}(z+d)}\right), \\
f_{+z}= & \left(a_{1+} e^{k_{+z}(z-d)}+a_{2+} e^{-k_{+z}(z+d)}\right) J_{l},
\end{aligned}
$$

and

$$
\begin{aligned}
f_{-r}= & \left(\frac{\mathrm{i} a_{1-}}{k_{r}}\left(\frac{l}{r} \frac{-k_{-}}{k_{r}} J_{l}-\mathrm{i} k_{-z} J_{l}^{\prime}\right) e^{k_{-z}(z-d)}\right) \\
& +\left(\frac{\mathrm{i} a_{2-}}{k_{r}}\left(\frac{l-\frac{-k_{-}}{r} J_{r}}{k_{l}}+\mathrm{i} k_{-z} J_{l}^{\prime}\right) e^{-k_{-z}(z+d)}\right),
\end{aligned}
$$




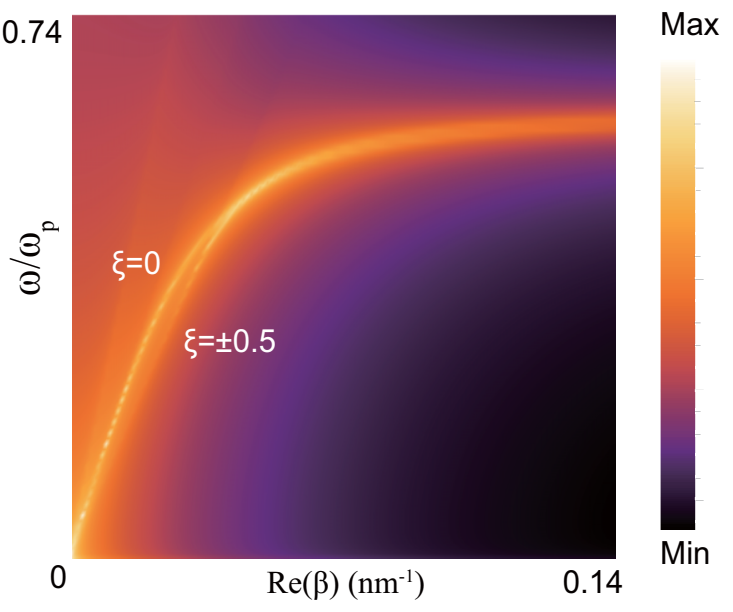

FIG. 6. Nonchiral versus chiral dispersions.

$$
\begin{aligned}
f_{-\varphi}= & \left(-\frac{a_{1-}}{k_{r}^{2}}\left(-\mathrm{i} k_{-z} \frac{l}{r} J_{l}+k_{r}\left(-k_{-}\right) J_{l}^{\prime}\right) e^{k_{-z}(z-d)}\right) \\
& +\left(-\frac{a_{2-}}{k_{r}^{2}}\left(\mathrm{i} k_{-z} \frac{l}{r} J_{l}+k_{r}\left(-k_{-}\right) J_{l}^{\prime}\right) e^{-k_{-z}(z+d)}\right), \\
f_{-z}= & \left(a_{1-} e^{k_{-z}(z-d)}+a_{2-} e^{-k_{-z}(z+d)}\right) J_{l} .
\end{aligned}
$$

The electromagnetic fields for a plasmonic OV (when $z=$ $0)$ can be derived as

$$
\begin{aligned}
& E_{r}=\frac{1}{2}\left[\frac { \mathrm { i } l } { r } \frac { J _ { l } } { k _ { r } ^ { 2 } } \left(a_{1+} k_{+} e^{k_{+z}(z-d)}+a_{2+} k_{+} e^{-k_{+z}(z+d)}\right.\right. \\
& \left.-a_{1-} k_{-} e^{+k_{-z}(z-d)}-a_{2-} k_{-} e^{-k_{-z}(z+d)}\right) \\
& +\frac{-J_{l}^{\prime}}{k_{r}}\left(-a_{1+} k_{+z} e^{k_{+z}(z-d)}+a_{2+} k_{+z} e^{-k_{+z}(z+d)}\right. \\
& \left.\left.-a_{1-} k_{-z} e^{k_{-z}(z-d)}+a_{2-} k_{-z} e^{-k_{-z}(z+d)}\right)\right], \\
& E_{\varphi}=\frac{1}{2}\left[\frac { - \mathrm { i } l } { r } \frac { J _ { l } } { k _ { r } ^ { 2 } } \left(-a_{1+} k_{+z} e^{k_{+z}(z-d)}+a_{2+} k_{+z} e^{-k_{+z}(z+d)}\right.\right. \\
& \left.-a_{1-} k_{-z} e^{k_{-z}(z-d)}+a_{2-} k_{-z} e^{-k_{-z}(z+d)}\right) \\
& +\frac{-J_{l}^{\prime}}{k_{r}}\left(a_{1+} k_{+} e^{k_{+z}(z-d)}+a_{2+} k_{+} e^{-k_{+z}(z+d)}\right. \\
& \left.\left.-a_{1-} k_{-} e^{k_{-z}(z-d)}-a_{2-} k_{-} e^{-k_{-z}(z+d)}\right)\right] \text {, } \\
& E_{z}=\frac{1}{2} J_{l}\left[a_{1+} e^{k_{+z}(z-d)}+a_{2+} e^{-k_{+z}(z+d)}\right. \\
& \left.+a_{1-} e^{k_{-z}(z-d)}+a_{2-} e^{-k_{-z}(z+d)}\right] \text {, } \\
& H_{r}=\frac{1}{2 \eta}\left[\frac { l } { r } \frac { J _ { l } } { k _ { r } ^ { 2 } } \left(a_{1+} k_{+} e^{k_{+z}(z-d)}+a_{2+} k_{+} e^{-k_{+z}(z+d)}\right.\right. \\
& \left.+a_{1-} k_{-} e^{k_{-z}(z-d)}+a_{2-} k_{-} e^{-k_{-z}(z+d)}\right) \\
& +\mathrm{i} \frac{J_{l}^{\prime}}{k_{r}}\left(-a_{1+} k_{+z} e^{k_{+z}(z-d)}+a_{2+} k_{+z} e^{-k_{+z}(z+d)}\right. \\
& \left.\left.+a_{1-} k_{-z} e^{k_{-z}(z-d)}-a_{2-} k_{-z} e^{-k_{-z}(z+d)}\right)\right] \text {, }
\end{aligned}
$$

$$
\begin{aligned}
H_{\varphi}= & \frac{1}{2 \eta}\left[-\frac{l}{r} \frac{J_{l}}{k_{r}^{2}}\left(-a_{1+} k_{+z} e^{+k_{+z}(z-d)}+a_{2+} k_{+z} e^{-k_{+z}(z+d)}\right.\right. \\
& \left.+a_{1-} k_{-z} e^{+k_{-z}(z-d)}-a_{2-} k_{-z} e^{-k_{-z}(z+d)}\right) \\
& +\mathrm{i} \frac{J_{l}^{\prime}}{k_{r}}\left(a_{1+} k_{+} e^{k_{+z}(z-d)}+a_{2+} k_{+} e^{-k_{+z}(z+d)}\right. \\
& \left.\left.+a_{1-} k_{-} e^{k_{-z}(z-d)}+a_{2-} k_{-} e^{-k_{-z}(z+d)}\right)\right] \\
& \\
H_{z}= & \frac{1}{2 \eta}\left(-\mathrm{i} J_{l}\right)\left[a_{1+} e^{k_{+z}(z-d)}+a_{2+} e^{-k_{+z}(z+d)}\right. \\
& \left.-a_{1-} e^{k_{-z}(z-d)}-a_{2-} e^{-k_{-z}(z+d)}\right] .
\end{aligned}
$$

In the top metal $(z>d)$,

$$
\begin{aligned}
E_{r} & =\left[\frac{-k_{z}}{k_{r}} B J_{l}^{\prime}-\frac{\omega \mu}{k_{r}^{2}} \frac{l}{r} C J_{l}\right] e^{-k_{z}(z-d)}, \\
E_{\varphi} & =\left[-\frac{\mathrm{i} k_{z}}{k_{r}^{2}} \frac{l}{r} B J_{l}-\frac{\mathrm{i} \omega \mu}{k_{r}} C J_{l}^{\prime}\right] e^{-k_{z}(z-d)}, \\
E_{z} & =B J_{l} e^{-k_{z}(z-d)},
\end{aligned}
$$

and

$$
\begin{aligned}
H_{r} & =\left[\frac{\omega \varepsilon_{m}}{k_{r}^{2}} \frac{l}{r} B J_{l}-\frac{k_{z}}{k_{r}} C J_{l}^{\prime}\right] e^{-k_{z}(z-d)}, \\
H_{\varphi} & =\left[\frac{\mathrm{i} \omega \varepsilon_{m}}{k_{r}} B J_{l}^{\prime}-\frac{\mathrm{i} k_{z}}{k_{r}^{2}} \frac{l}{r} C J_{l}\right] e^{-k_{z}(z-d)}, \\
H_{z} & =C J_{l} e^{-k_{z}(z-d)} .
\end{aligned}
$$

In the bottom metal $(z<-d)$,

$$
\begin{aligned}
E_{r} & =\left[\frac{k_{z}}{k_{r}} D J_{l}^{\prime}-\frac{\omega \mu}{k_{r}^{2}} \frac{l}{r} E J_{l}\right] e^{k_{z}(z+d)}, \\
E_{\varphi} & =\left[\frac{\mathrm{i} k_{z}}{k_{r}^{2}} \frac{l}{r} D J_{l}-\frac{\mathrm{i} \omega \mu}{k_{r}} E J_{l}^{\prime}\right] e^{k_{z}(z+d)}, \\
E_{z} & =D J_{l} e^{k_{z}(z+d)},
\end{aligned}
$$

and

$$
\begin{aligned}
& H_{r}=\left[\frac{\omega \varepsilon_{m}}{k_{r}^{2}} \frac{l}{r} D J_{l}+\frac{k_{z}}{k_{r}} E J_{l}^{\prime}\right] e^{k_{z}(z+d)}, \\
& H_{\varphi}=\left[\frac{\mathrm{i} \omega \varepsilon_{m}}{k_{r}} D J_{l}^{\prime}+\frac{\mathrm{i} k_{z}}{k_{r}^{2}} \frac{l}{r} E J_{l}\right] e^{k_{z}(z+d)}, \\
& H_{z}=E J_{l} e^{k_{z}(z+d)}
\end{aligned}
$$

Subsequently, the SAM of the plasmonic OV can be derived as in Eq. (2) in the main text. The boundary conditions of $E_{r}, E_{\varphi}, H_{r}$, and $H_{\varphi}$ at $z=-d$ and $z=+d$ leads to an $8 \times 8$ matrix as a function of the coefficients $a_{1 \pm}, a_{2 \pm}, B, C, D$, and $E$. Also, comparison of the dispersion curves for nonchiral 


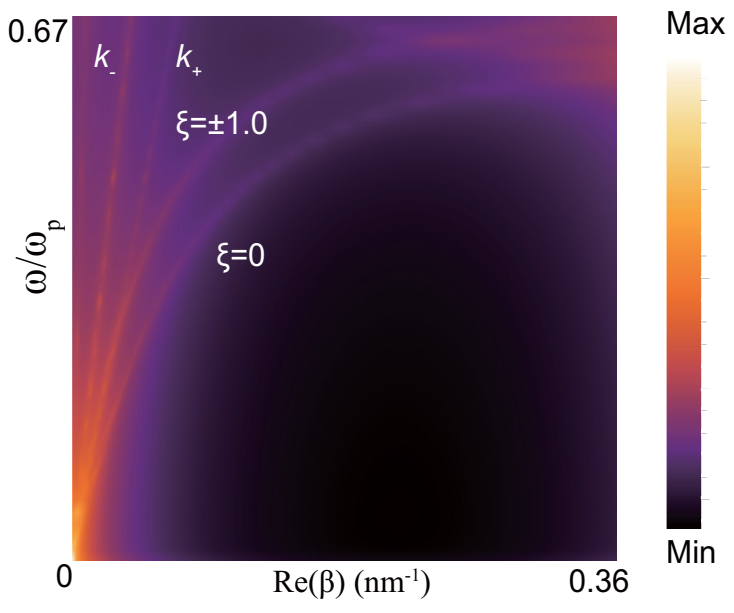

FIG. 7. Nonchiral versus chiral dispersions.

and chiral plasmonic OVs is given in Fig. 7 (here we choose a large chirality parameter to better show the discrepancy).

\section{APPENDIX C: ENERGY FLUX DENSITY FOR \\ PLASMONIC OPTICAL VORTEX AT A METAL-CHIRAL-METAL STRUCTURE}

The energy flux density in the chiral medium is defined as $\mathbf{P}=\operatorname{Re}\left(\mathbf{E}^{*} \times \mathbf{H}\right) / 2$. By replacing $\mathbf{E}$ and $\mathbf{H}$ with those listed in Sec. B, we obtain

$$
\begin{aligned}
P_{r}= & \frac{1}{4 \eta} \frac{l}{r} \frac{J_{l}^{2}}{k_{r}^{2}}\left[-a_{1+}^{2} k_{+z} e^{2 k_{+z}(z-d)}+a_{2+}^{2} k_{+z} e^{-2 k_{+z}(z+d)}\right. \\
& \left.a_{1-}^{2} k_{-z} e^{2 k_{-z}(z-d)}-a_{2-}^{2} k_{-z} e^{-2 k_{-z}(z+d)}\right], \\
P_{\varphi}= & \frac{1}{4 \eta} \frac{l}{r} \frac{J_{l}^{2}}{k_{r}^{2}}\left[\left(a_{1+}^{2} k_{+} e^{2 k_{+z}(z-d)}+a_{2+}^{2} k_{+} e^{-2 k_{+z}(z+d)}\right)\right. \\
& +\left(a_{1-}^{2} k_{-} e^{2 k_{-z}(z-d)}+a_{2-}^{2} k_{-} e^{-2 k_{-z}(z+d)}\right) \\
& \left.+2 a_{1+} a_{2+} e^{-2 k_{+z} d} k_{+}+2 a_{1-} a_{2-} e^{-2 k_{-z} d} k_{-}\right], \\
P_{z}= & \frac{1}{4 \eta} \frac{l}{r} \frac{J_{l} J_{l}^{\prime}}{k_{r}^{3}} \\
\times & {\left[\left(a_{1+}^{2} e^{2 k_{+z}(z-d)}+a_{2+}^{2} e^{-2 k_{+z}(z+d)}-2 a_{1+} a_{2+} e^{-2 k_{+z} d}\right) k_{+z}^{2}\right.} \\
- & \left(a_{1-}^{2} e^{2 k_{-z}(z-d)}+a_{2-}^{2} e^{-2 k_{-z}(z+d)}-2 a_{1-} a_{2-} e^{-2 k_{-z} d}\right) k_{-z}^{2} \\
+ & {\left[\left(a_{1+}^{2} e^{2 k_{+z}(z-d)}+a_{2+}^{2} e^{-2 k_{+z}(z+d)}+2 a_{1+} a_{2+} e^{-2 k_{+z} d}\right) k_{+}^{2}\right.} \\
- & \left.\left(a_{1-}^{2} e^{2 k_{-z}(z-d)}+a_{2-}^{2} e^{-2 k_{-z}(z+d)}+2 a_{1-} a_{2-} e^{-2 k_{-z} d}\right) k_{-}^{2}\right] .
\end{aligned}
$$

\section{APPENDIX D: DEPENDENCE OF THE SKYRMION'S SAM VECTOR PROFILES ON THE CHIRAL FILM'S THICKNESS}

As we mentioned in the main text, when the film thickness is very thin, the strong coupling between the plasmonic OVs at the top and bottom surfaces leads to a reverse $S_{\varphi}$, compared to that of a single interface plasmonic OV, under the same chirality $\xi$. When the film thickness increases, the coupling
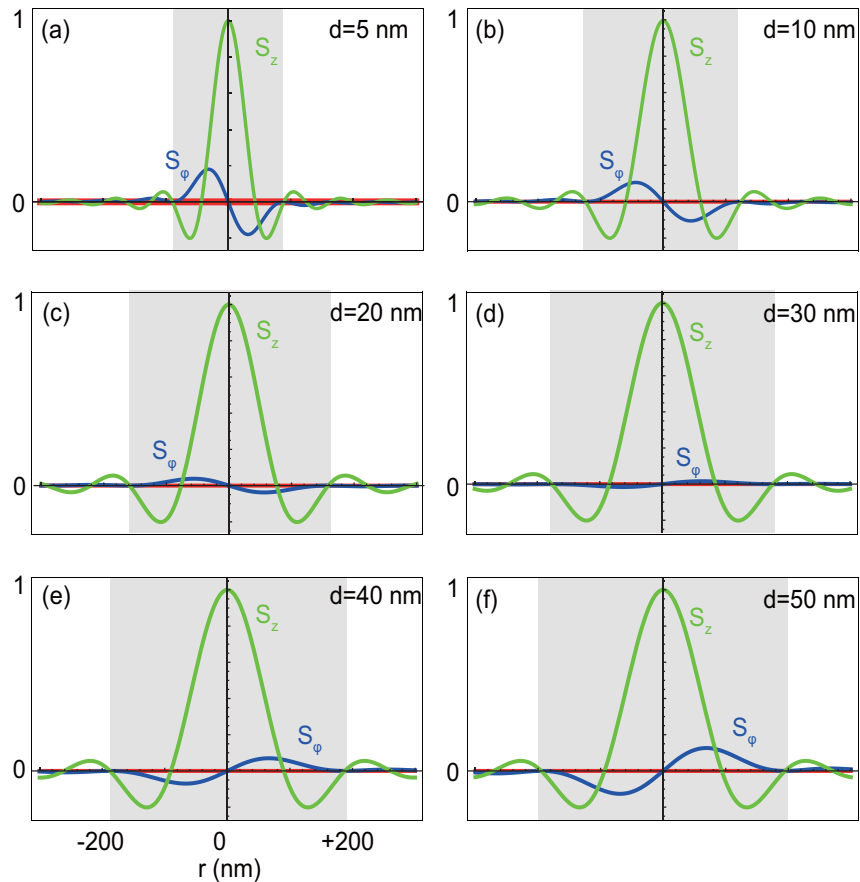

FIG. 8. Profiles of the SAM vectors under various chiral film thickness $(2 d=10 \mathrm{~nm}, 20 \mathrm{~nm}, 40 \mathrm{~nm}, 60 \mathrm{~nm}, 80 \mathrm{~nm}$, and $100 \mathrm{~nm}$, respectively). The chirality $\xi$ is set to +0.15 for all cases.

becomes weak and the behavior reduces to the one similar with single interface plasmonic OV, as shown in Fig. 8.

\section{APPENDIX E: BLOCH PHOTONIC SKYRMIONS IN AN ASYMMETRIC METAL-CHIRAL-METAL STRUCTURE}

Here we choose $\gamma=1.08 \times 10^{14} \mathrm{rad} / \mathrm{sec}, \omega_{p m}=1.35 \times$ $10^{16} \mathrm{rad} / \mathrm{sec}$ for Au as the top metal, whereas $\gamma=1.5 \times 10^{14}$ $\mathrm{rad} / \mathrm{sec}, \omega_{p m}=1 \times 10^{16} \mathrm{rad} / \mathrm{sec}$ for a different metallic material with negative permittivity as the bottom metal. The evolution of the skyrmion structure's helicity angle along the chiral film is given in Fig. 9. We recall in the main text that a Bloch photonic skyrmion exists in the center of a symmetric plasmonic structure while in an asymmetric structure, a Bloch photonic skyrmion still exists, but is not in the center.

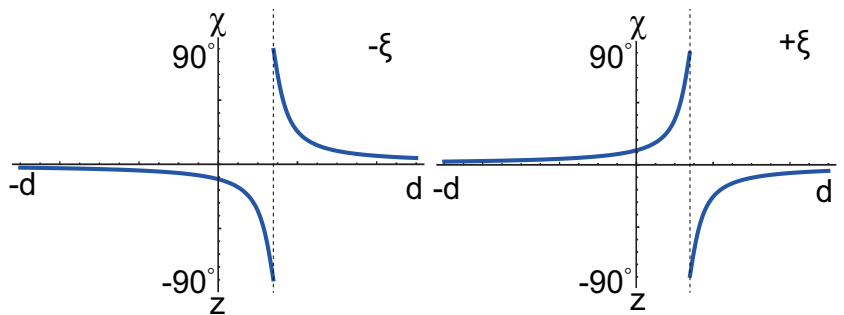

FIG. 9. Diagram for the evolution of the helicity angle of the skyrmion's spin texture as a function of the chiral film thickness $(10 \mathrm{~nm})$. 
[1] A. Bogdanov and D. Yablonskii, Thermodynamically stable "vortices" in magnetically ordered crystals. The mixed state of magnets, Zh. Eksp. Teor. Fiz 95, 178 (1989) [Sov. Phys. JETP 68, 101 (1989)].

[2] A. Bogdanov and A. Hubert, Thermodynamically stable magnetic vortex states in magnetic crystals, J. Magn. Magn. Mater. 138, 255 (1994).

[3] S. S. P. Parkin, M. Hayashi, and L. Thomas, Magnetic domainwall racetrack memory, Science 320, 190 (2008).

[4] T. Kurumaji, T. Nakajima, M. Hirschberger, A. Kikkawa, Y. Yamasaki, H. Sagayama, H. Nakao, Y. Taguchi, T.-h. Arima, and Y. Tokura, Skyrmion lattice with a giant topological Hall effect in a frustrated triangular-lattice magnet, Science 365, 914 (2019).

[5] D. Foster, C. Kind, P. J. Ackerman, J.-S. B. Tai, M. R. Dennis, and I. I. Smalyukh, Two-dimensional skyrmion bags in liquid crystals and ferromagnets, Nat. Phys. 15, 655 (2019).

[6] A. J. Hess, G. Poy, Jung-Shen B. Tai, S. Žumer, and I. I. Smalyukh, Control of Light by Topological Solitons in Soft Chiral Birefringent Media, Phys. Rev. X 10, 031042 (2020).

[7] T. Yokouchi, F. Kagawa, M. Hirschberger, Y. Otani, N. Nagaosa, and Y. Tokura, Two-dimensional skyrmion bags in liquid crystals and ferromagnets, Nature (London) 586, 232 (2020).

[8] S. Mühlbauer, B. Binz, F. Jonietz, C. Pfleiderer, A. Rosch, A. Neubauer, R. Georgii, and P. Böni, Skyrmion lattice in a chiral magnet, Science 323, 915 (2009).

[9] X. Z. Yu, Y. Onose, N. Kanazawa, J. H. Park, J. H. Han, Y. Matsui, N. Nagaosa, and Y. Tokura, Real-space observation of a two-dimensional skyrmion crystal, Nature (London) 465, 901 (2010).

[10] S. Heinze, K. von Bergmann, M. Menzel, J. Brede, A. Kubetzka, R. Wiesendanger, G. Bihlmayer, and S. Blügel, Spontaneous atomic-scale magnetic skyrmion lattice in two dimensions, Nat. Phys. 7, 713 (2011).

[11] N. Nagaosa and Y. Tokura, Topological properties and dynamics of magnetic skyrmions, Nat. Nanotechnol. 8, 899 (2013).

[12] I. Kézsmárki, S. Bordács, P. Milde, E. Neuber, L. M. Eng, J. S. White, H. M. Rø nnow, C. D. Dewhurst, M. Mochizuki, K. Yanai, H. Nakamura, D. Ehlers, V. Tsurkan, and A. Loidl, NÃ@el-type skyrmion lattice with confined orientation in the polar magnetic semiconductor GaV4S8, Nat. Mater. 14, 1116 (2015).

[13] R. Wiesendanger, Nanoscale magnetic skyrmions in metallic films and multilayers: A new twist for spintronics, Nat. Rev. Mater. 1, 16044 (2016).

[14] A. Fert, N. Reyren, and V. Cros, Magnetic skyrmions: Advances in physics and potential applications, Nat. Rev. Mater. 2, 17031 (2017).

[15] A. N. Bogdanov and C. Panagopoulos, Physical foundations and basic properties of magnetic skyrmions, Nat. Rev. Phys. 2, 492 (2020).

[16] S. Tsesses, E. Ostrovsky, K. Cohen, B. Gjonaj, N. H. Lindner, and G. Bartal, Optical skyrmion lattice in evanescent electromagnetic fields, Science 361, 993 (2018).

[17] L. Du, A. Yang, A. V. Zayats, and X. Yuan, Deepsubwavelength features of photonic skyrmions in a confined electromagnetic field with orbital angular momentum, Nat. Phys. 15, 650 (2019).
[18] T. J. Davis, D. Janoschka, P. Dreher, B. Frank, F.-J. Meyer zu Heringdorf, and H. Giessen, Ultrafast vector imaging of plasmonic skyrmion dynamics with deep subwavelength resolution, Science 368, eaba6415 (2020).

[19] S. Tsesses, K. Cohen, E. Ostrovsky, B. Gjonaj, and G. Bartal, Spinorbit interaction of light in plasmonic lattices, Nano Lett. 19, 4010 (2019).

[20] C. Bai, J. Chen, Y. Zhang, D. Zhang, and Q. Zhan, Dynamic tailoring of an optical skyrmion lattice in surface plasmon polaritons, Opt. Express 28, 10320 (2020).

[21] X.-G. Wang, L. Chotorlishvili, N. Arnold, V. K. Dugaev, I. Maznichenko, J. Barnaś, P. A. Buczek, S. S. P. Parkin, and A. Ernst, Plasmonic Skyrmion Lattice Based on the Magnetoelectric Effect, Phys. Rev. Lett. 125, 227201 (2020).

[22] S. A. Maier, Plasmonics: Fundamentals and Applications (Springer, New York, NY, 2007).

[23] K. Y. Bliokh, D. Smirnova, and F. Nori, Quantum spin Hall effect of light, Science 348, 1448 (2015).

[24] K. Y. Bliokh, A. Y. Bekshaev, and F. Nori, Extraordinary momentum and spin in evanescent waves, Nat. Commun. 5, 3300 (2014).

[25] A. Aiello, N. Lindlein, C. Marquardt, and G. Leuchs, Transverse Angular Momentum and Geometric Spin Hall Effect of Light, Phys. Rev. Lett. 103, 100401 (2009).

[26] K. Y. Bliokh and F. Nori, Transverse spin of a surface polariton, Phys. Rev. A 85, 061801(R) (2012).

[27] K. Y. Bliokh and F. Nori, Transverse and longitudinal angular momenta of light, Phys. Rep. 592, 1 (2015).

[28] T. V. Mechelen and Z. Jacob, Universal spin-momentum locking of evanescent waves, Optica 3, 118 (2016).

[29] N. Engheta and P. Pelet, Modes in chirowaveguides, Opt. Lett. 14, 593 (1989).

[30] I. V. Lindell, A. H. Sihvola, S. A. Tretyakov, and A. J. Viitanen, Electromagnetic Waves in Chiral and Bi-isotropic Media (Artech House, London, 1994).

[31] C. F. Bohren, Light scattering by an optically active sphere, Chem. Phys. Lett. 29, 458 (1974).

[32] C. Kelly, L. Khosravi Khorashad, N. Gadegaard, L. D. Barron, A. O. Govorov, A. S. Karimullah, and M. Kadodwala, Controlling metamaterial transparency with superchiral fields, ACS Photonics 5, 535 (2018).

[33] F. N. Rybakov, A. B. Borisov, and A. N. Bogdanov, Threedimensional skyrmion states in thin films of cubic helimagnets, Phys. Rev. B 87, 094424 (2013).

[34] S. A. Meynell, M. N. Wilson, H. Fritzsche, A. N. Bogdanov, and T. L. Monchesky, Surface twist instabilities and skyrmion states in chiral ferromagnets, Phys. Rev. B 90, 014406 (2014).

[35] A. O. Leonov, Y. Togawa, T. L. Monchesky, A. N. Bogdanov, J. Kishine, Y. Kousaka, M. Miyagawa, T. Koyama, J. Akimitsu, T. Koyama, K. Harada, S. Mori, D. McGrouther, R. Lamb, M. Krajnak, S. McVitie, R. L. Stamps, and K. Inoue, Chiral Surface Twists and Skyrmion Stability in Nanolayers of Cubic Helimagnets, Phys. Rev. Lett. 117, 087202 (2016).

[36] S. L. Zhang, G. van der Laan, W. W. Wang, A. A. Haghighirad, and T. Hesjedal, Direct Observation of Twisted Surface Skyrmions in Bulk Crystals, Phys. Rev. Lett. 120, 227202 (2018). 
[37] P. Shi, L. Du, C. Li, A. Zayats, and X. Yuan, Transverse spin dynamics in structured electromagnetic guided waves, Proc. Natl. Acad. Sci. USA 118, e2018816118 (2021).

[38] P. Shi, L. Du, and X. Yuan, Strong spin-orbit interaction of photonic skyrmions at the general optical interface, Nanophotonics 9, 4619 (2020).

[39] C. Moreau-Luchaire, C. Moutafis, N. Reyren, J. Sampaio, C. A. F. Vaz, N. Van Horne, K. Bouzehouane, K. Garcia, C. Deranlot, P. Warnicke, P. Wohlhüter, J.-M. George, M. Weigand, J. Raabe, V. Cros, and A. Fert, Additive interfacial chiral interaction in multilayers for stabilization of small indi- vidual skyrmions at room temperature, Nat. Nanotechnol. 11, 444 (2016).

[40] A. Soumyanarayanan, M. Raju, A. L. Gonzalez Oyarce, A. K. C. Tan, M.-Y. Im, A. P. Petrović, P. Ho, K. H. Khoo, M. Tran, C. K. Gan, F. Ernult, and C. Panagopoulos, Tunable room-temperature magnetic skyrmions in $\mathrm{Ir} / \mathrm{Fe} / \mathrm{Co} / \mathrm{Pt}$ multilayers, Nat. Mater. 16, 898 (2017).

[41] P. Lodahl, S. Mahmoodian, S. Stobbe, A. Rauschenbeutel, P. Schneeweiss, J. Volz, H. Pichler, and P. Zoller, Chiral quantum optics, Nature (London) 541, 473 (2017).

[42] F. Träger, Springer Handbook of Lasers and Optics (Springer, New York, 2012). 